\title{
Paraneoplastic Sensory Neuronopathy and Spontaneous Regression of Small Cell Lung Cancer
}

\author{
Sharlene Gill, Nevin Murray, Josep Dalmau and Brian Thiessen
}

\begin{abstract}
Background: Spontaneous tumour regression in small cell lung cancer has previously been suggested in patients with paraneoplastic neurologic syndromes. Rare documentation of this event has occurred in the literature. Case Report: The authors report a patient with anti-Hu associated paraneoplastic sensory neuronopathy who had a spontaneous regression of her small cell lung cancer. Conclusions: This case supports the hypothesis that anti-Hu neurologic syndromes are the consequence of a misdirected immune response to small cell tumours.
\end{abstract}

RÉSUMÉ: Neuropathie sensitive paranéoplasique et régression spontanée du cancer du poumon à petites cellules. Introduction: Une régression spontanée du cancer du poumon à petites cellules a déjà été observée chez des patients présentant des syndromes neurologiques paranéoplasiques. De rares cas ont été décrits dans la littérature. Étude de cas: Les auteurs rapportent le cas d'un patient présentant une neuropathie sensitive paranéoplasique avec anticorps anti-Hu chez qui un cancer du poumon à petites cellules a régressé spontanément. Conclusions: Ce cas appuie l'hypothèse selon laquelle les syndromes neurologiques anti-Hu sont la conséquence d'une réponse immunitaire aberrante à des tumeurs à petites cellules.

Can. J. Neurol. Sci. 2003; 30: 269-271

A 43-year-old woman was diagnosed with small cell lung cancer and a progressive neurologic paraneoplastic syndrome. During evaluation a remarkable regression of her lung cancer was noted prior to treatment. High serum titres of anti-Hu antibody were present which reacted against both central nervous system neuronal nuclei and tumor cells derived from the patient. We propose that the regression of lung cancer in this case was a direct result of a host immune response directed at both the cancer and the nervous system.

\section{Case Report}

A previously well, uniparous 43-year-old caucasian woman was transferred to the British Columbia Cancer Agency (N.M.) in October 2000 for evaluation of progressive neurologic dysfunction and a recent diagnosis of small cell lung cancer (SCLC). She had presented in August 2000 with a 25-pack year smoking history and two months of dyspnea, cough, weight loss, fatigue and chest X-ray (CXR) findings of a right hilar mass with paratracheal adenopathy (Figure 1A). Physical examination revealed a $1.5 \mathrm{~cm}$ right supraclavicular node which, on biopsy, confirmed SCLC.

Following her biopsy, she developed extremity weakness associated with gait ataxia and dysarthria. Bladder function was impaired with saddle dysesthesia. On examination, she had mild generalized weakness with reduced vibration and proprioception in all distal extremities and absent patellar and ankle reflexes. Her plantar responses were downgoing. Dysdiadochokinesia was observed in the left upper extremity. Examination of cranial nerves was normal.

No abnormalities were identified on gadolinium-enhanced magnetic resonance imaging of the brain and spine. Cerebrospinal fluid (CSF) analysis revealed a lymphocytic pleocytosis (WBC $30 \times 10^{6} / \mathrm{L}, 84 \%$ lymphocytes) and increased protein $(2325 \mathrm{mg} / \mathrm{L})$ with a prominent $\mathrm{IgG}$ fraction; CSF cytology was negative. High serum titres of anti-Hu antibodies (Hu- $\mathrm{Ab}$; upper serum dilution with immunoblot reactivity: $1: 512,000)$ confirmed a paraneoplastic neurologic syndrome in association with limited-stage SCLC. These antibodies reacted strongly against nuclear and cytoplasmic antigens of the patient's tumor on immunohistochemistry (Figure 2). A chest X-ray was repeated prior to commencement of chemotherapy with cisplatin and etoposide. Remarkably, there was almost complete spontaneous resolution of her previous right hilar and paratracheal abnormality (Figure 1B). She proceeded to receive dexamethasone and four cycles of chemotherapy

From the Division of Medical Oncology, British Columbia Cancer Agency, Vancouver BC (SG, NM, BT), Division of Neurology, Vancouver General Hospital, Vancouver, BC (BT), Canada and Department of Neurology, University of Arkansas for Medical Sciences, Little Rock, Arkansas, USA (JD).

Received August 22, 2002. AcCePted in final form February 20, 2003. Reprint requests to: Brian Thiessen, Division of Medical Oncology, British Columbia Cancer Agency, 600 West 10th Avenue, Vancouver, BC V5Z 4E6 Canada. 


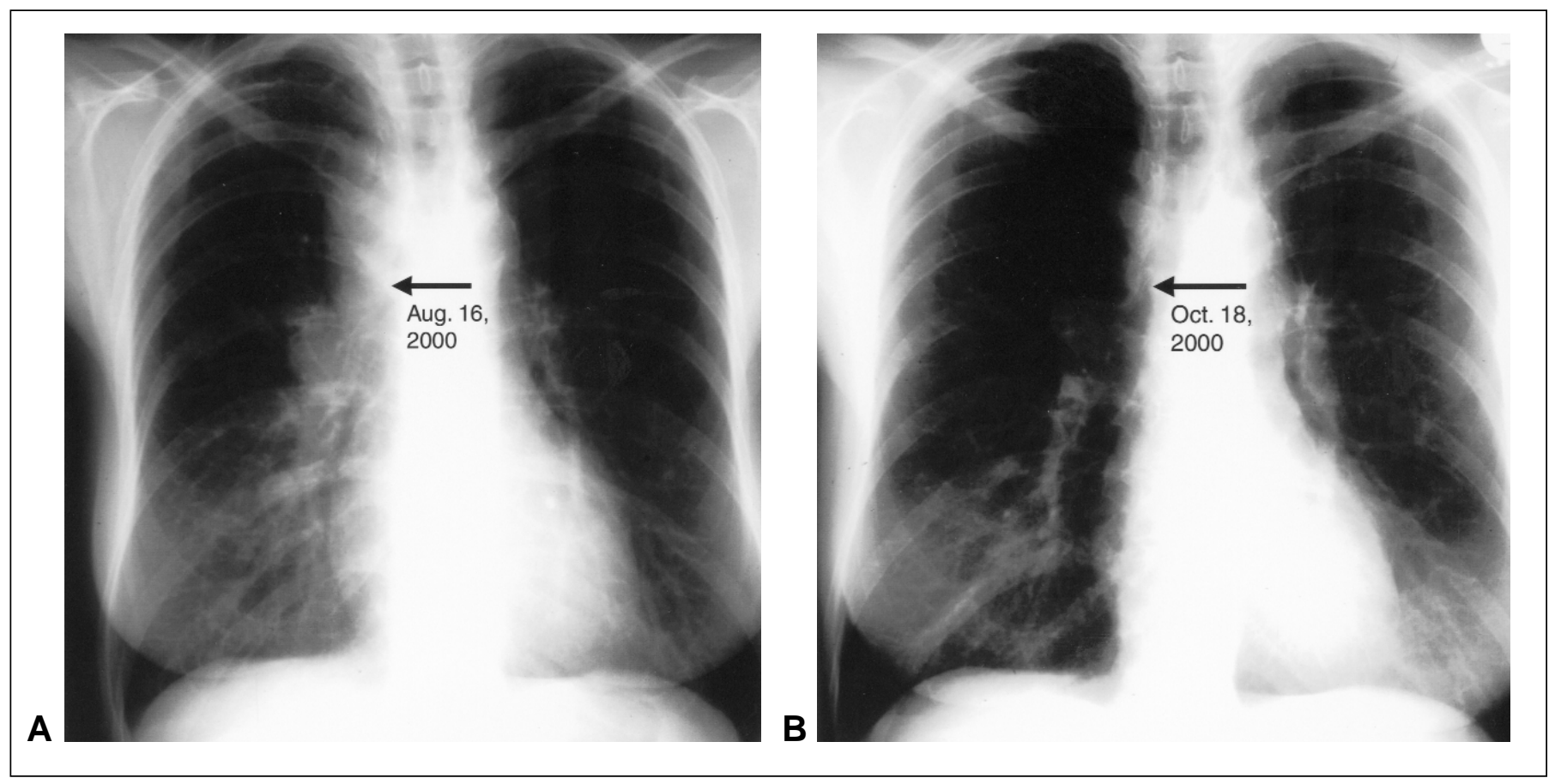

Figure 1: Left image (August 16, 2000) demonstrates a right hilar mass (arrow) present at diagnosis. Right image (October 18, 2000) performed prior to initiation of chemotherapy, shows spontaneous resolution of the right hilar mass (arrow).

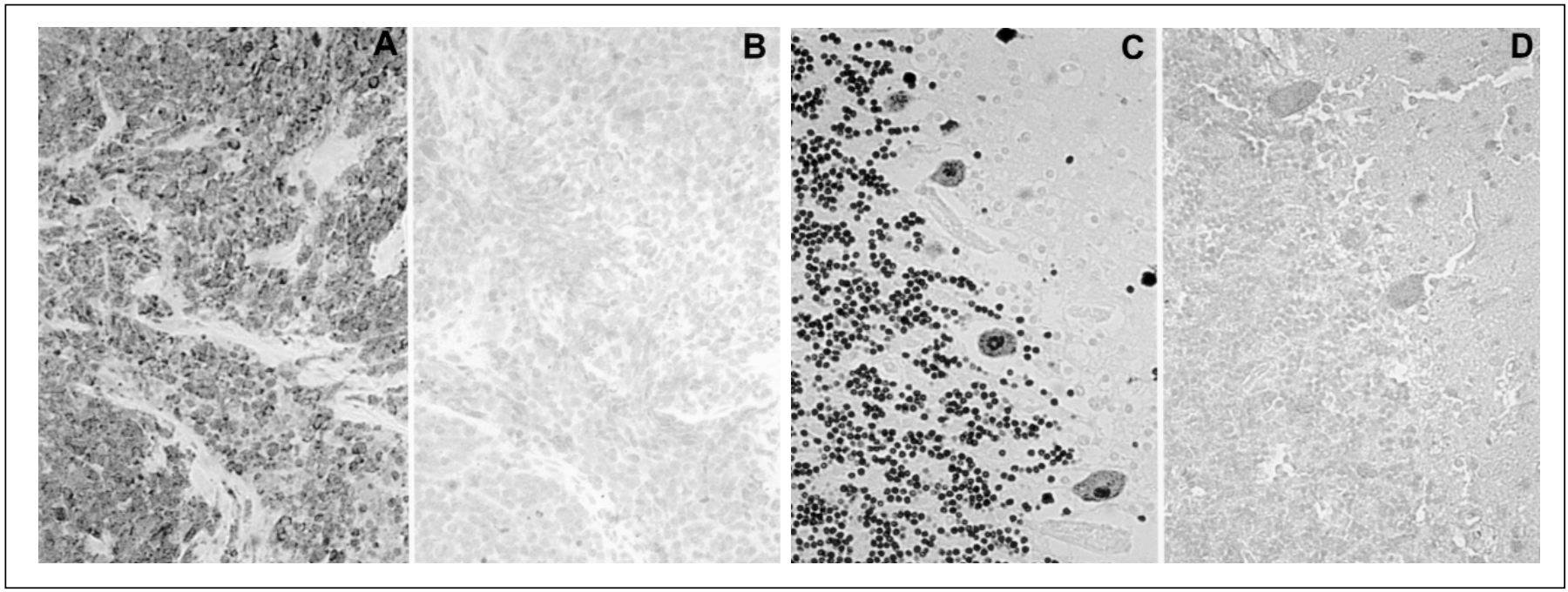

Figure 2: Panel A and B: Immunohistochemical analysis of the patient's tumor incubated with IgG isolated from the patient's serum (A) and IgG isolated from a normal individual $(B)$. There is intense tumor reactivity with nuclear and cytoplasmic staining of the tumour with the patient's serum; similar reactivity was obtained with anti-Hu IgG from another patient (data not shown). The immunohistochemical analysis was performed using a technique previously reported, which includes isolation of the patient's IgG and subsequent labeling with biotin. ${ }^{6}$ Both sections have been mildly counterstained with hematoxylin.

Panels $C$ and D: Immunohistochemical analysis on rat brain sections using the patient's serum IgG and the serum IgG of a normal individual (D) demonstrating nuclear staining of the neuronal tissue. The specific neuronal reactivity with the patient's IgG corresponds with the anti-Hu antibody, confirmed in immunoblot analysis (data not shown). 
with thoracic irradiation. While her neurologic syndrome stabilized with a coincident decrease in $\mathrm{Hu}-\mathrm{Ab}$ serum titres $(1: 32,000)$, significant deficits persisted with ataxia, pseudo-athetoid movements of the upper extremities and diffuse, multisegmental sensory loss for all modalities. Subsequent electrophysiologic studies were consistent with a sensory neuronopathy with complete absence of sensory nerve action potentials and normal motor nerve conduction. At the time of discharge, the patient's tumor was in complete remission. She remained wheelchair dependent and required chronic analgesics for neuropathic pain.

\section{Discussion}

In patients with SCLC the development of paraneoplastic encephalomyelitis and sensory neuronopathy (PEM/PSN) is usually associated with elevated titres of $\mathrm{Hu}-\mathrm{Ab}$. These antibodies are markers of an immune response to a family of neuronal-specific RNA binding proteins (Hu antigens) expressed by the tumor. ${ }^{1}$ The detection of serum $\mathrm{Hu}-\mathrm{Ab}$ at the time of tumor diagnosis is usually associated with limited stage disease and, even at low titres, they serve as a predictor of tumor response to treatment and longer survival. It has been postulated that the tumor expression of $\mathrm{Hu}$ antigens may enhance antitumoral immunity and increase chemosensitivity. ${ }^{2}$

A previous report of three patients with PEM/PSN suggested the occurrence of spontaneous tumor regression, but the study was confounded by limited evaluation. ${ }^{3}$ In this small series, spontaneous regression of a lung mass was reported in a single case but without pathological confirmation of SCLC. In the remaining two cases, sustained regression was documented after chemotherapy but anti-Hu antibodies were identified in only one of these two patients.

The previous speculation of spontaneous tumor regression is now confirmed with our case presentation of SCLC regression documented prior to chemotherapy. While the explicit mechanism of this regression remains uncertain, this case gives further evidence that PEM/PSN results from a tumor-induced immune response that is misdirected against nervous system antigens. The temporal association of spontaneous tumor regression with the development of neurologic symptoms, and the expression of $\mathrm{Hu}$ antigens by the tumor support this hypothesis. While treatment of the underlying SCLC may stabilize the paraneoplastic syndrome, long-term neurologic sequelae are common. ${ }^{4}$ Immunosuppression with plasmapheresis, corticosteroids, and intravenous immunoglobulin is of minimal benefit. While it is theoretically conceivable that immunosuppression may cancel any protective antitumor immune response mediated by neuronal antibodies, clinical series to-date have failed to demonstrate inferior SCLC outcomes among PEM/PSN patients treated with immunotherapy or systemic chemotherapy. ${ }^{4,5}$ Continued efforts are required to determine the optimal management for these patients. $^{7}$

\section{REFERENCES}

1. Budde-Steffen C, Anderson NE, Rosenblum MK, et al. Expression of an antigen in small cell lung carcinoma lines detected by antibodies from patients with paraneoplastic dorsal root gangliopathy. Cancer Res 1988;48:430-434.

2. Graus F, Dalmau J, Rene R et al. Anti-Hu antibodies in patients with small cell lung cancer - association with complete response to therapy and improved survival. J Clin Oncol 1997;15:2866-2872.

3. Darnell RB, DeAngelis LM. Regression of small-cell lung carcinoma in patients with paraneoplastic neuronal antibodies. Lancet 1993;341:21-22.

4. Keime-Guibert F, Graus F, Broet P, et al. Clinical outcome of patients with anti-Hu associated encephalomyelitis after treatment of the tumour. Neurology 1999;53(8):1719-1723.

5. Smitt PS, Grefkens J, deLeeuw B, et al. Survival and outcome in 73 anti-Hu positive patients with paraneoplastic encephalomyelitis/ sensory neuronopathy. J Neurol 2002; 249: 745-753.

6. Furneaux HM, Rosenblum MK, Dalmau J, et al. Selective expression of Purkinje-cell antigens in tumour tissue from patients with paraneoplastic cerebellar degeneration. N Engl J Med 1990;322:1844-1851.

7. Dalmau J, Graus F, Rosenblum MK, et al. Anti-Hu associated paraneoplastic encephalomyelitis/sensory neuronopathy-a clinical study of 71 patients. Medicine (Baltimore) 1992;71:59. 